\title{
Planar Imaging of Stagnant and Hydrodynamic Fluid using
}

\section{Miniaturized ECT Device}

\author{
Shahrulnizahani Mohammad Din ${ }^{1}$, Leow Pei Ling ${ }^{2 *}$, Nur Adila Mohd Razali² \\ Aizat Azmi², Jaysuman Puspanathan ${ }^{3}$, Chee pei Song ${ }^{4}$, Ruzairi Abdul Rahim ${ }^{5,2}$
}

\author{
${ }^{1}$ Research Management Center, Universiti Teknologi Malaysia, 81310 Skudai, Johor \\ ${ }^{2}$ Faculty of Electrical Engineering, Universiti Teknologi Malaysia, 81310 Skudai, Johor \\ ${ }^{3}$ Faculty of Bioscience and Medical Engineering, University Teknologi Malaysia, 81310 Skudai, Johor \\ ${ }^{4}$ Lee Kong Chian Fac. of Eng. \& Sci., Universiti Tunku Abdul Rahman, Bandar Sungai Long, 43000, Kajang, \\ ${ }^{5}$ Faculty of Electrical and Electronic Engineering, Universiti Tun Hussein Onn, 86400 Batu Pahat, Johor
}

\section{Received 6 June 2018; accepted 25 November 2018, available online 31 December 2018}

\begin{abstract}
:
This paper discusses the development of an on chip planar capacitance tomography (ECT) for stagnant and hydrodynamic multiphase fluid monitoring. The 8-electrode planar ECT device is developed based on Lab-on-chip (LOC) or microfluidic device concept. The ECT with LOC application is an improved design to allow ECT to be portable and small scaled measurement. To reconstruct images, ECT measures the permittivity variation within the sensing area. Eight-planar electrodes with dimension of $5.00 \mathrm{~mm} \times 2.08 \mathrm{~mm}$ (length $\times$ width) are fabricated on printed circuit board (PCB). These electrodes are aligned to circulate $16 \mathrm{~mm}$ diameter of sensing area. For stagnant sample, the design of the chamber is simpler from the hydrodynamic flow. The hydrodynamic flow consists with two inlets to allow multiphase flow testing. Fan beam projection technique is utilized with Linear Back Projection (LBP) algorithm to reconstruct images of the substance within the chamber. Time is an important item for hydrodynamic flow compared to stagnant sample to ensure that the data taken is useful to the user. For stagnant experiment, the ECT system able to produce image reconstruction for water - air, oil - water and yeast - glucose samples. For hydrodynamic flow, the test conducted for immiscible water - oil sample, and miscible water glucose solution is done. The results show the system produce the best result when one liquid is dominating the sensing area.
\end{abstract}

Keywords: Planar, ECT, static and hydrodynamic flow, miniature ECT

\section{Introduction}

Process Tomography (PT) is a technique that produces cross-sectional images of an enclosed pipeline [1]. The system operates without obstructing and affecting the flow inside it. These cross-sectional images within the pipeline system are obtained by using various types of sensing array. The images produced from sets of data obtained during the inspection. There are many types of PT in industrial and medical application such as Ultrasonic Tomography (UT), Electrical Resistance Tomography (ERT), Positron emission tomography (PET), Electrical Impedance Tomography (EIT) and Electrical Capacitance Tomography (ECT) $[1,7,8]$. The selected PT type is based on application and purpose of the analysis. Among items to be considered are the environment, the material inside the inspection area, the size of the sample and the sensing element.

Electrical Capacitance Tomography (ECT) is one of the electrical tomography besides EIT and ERT. ECT allows user to inspect the flow distribution inside a closed vessel by detecting the permittivity variation. Although ECT suffers from soft-field effect, it remains prominent system as it provide low cost setup, fast response and radiation free system [1]. Apart from the drawback, the ECT soft-field measurement is sensitive in multiphase of different permittivity [2]. In addition, ECT provides noninvasive and non-intrusive measurements, which allows the test material flows naturally. The ECT system is divided into two components; hardware and software. The hardware component comprises of sensors and data control system. Signal reconstruction system and the display and interpretation facilities are the software component used in the system for imaging and data processing [3].

The basic tomography setup is often bulk and sophisticated, which contribute to high material and setup cost. The bulky size also limits its usage for in-situ application. There are many study conducted for 
miniature tomography to cater various applications such as teaching instruments and onsite inspection. The miniature tomography adopted lab-on-chip (LOC) application which able to provide high throughput and low cost setup [4]. In addition, LOC application uses small amount of sample. At the same time applies small quantity of reagents or chemical solution. LOC application includes in biotechnology, medical diagnostics and environmental monitoring [5].

This paper discusses the ECT adopted LOC application for stagnant and hydrodynamic flow. For LOC platform, the configuration of the electrodes are arranged in a planar where all electrodes are at the base of the sensing area [4] where the electrode configuration is shown in Fig. 1.

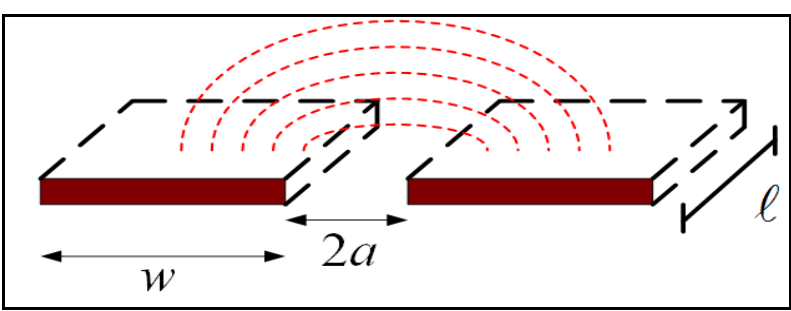

Fig. 1 Illustration of dimension and electrical field line formed by planar electrodes

The capacitance measurement for planar electrode configuration is defined as the measurement of charge between two plates (conductive electrode) inside the capacitor system. In miniaturized planar electrode sensor, the capacitance, $\mathrm{C}_{\mathrm{x}}$ is calculated based on equation (1) [4] where the field line of planar electrodes is formed above the electrodes when current is supplied to the excitation electrode.

$C_{\mathrm{X}}=\frac{2 \varepsilon_{r} \varepsilon_{o} \ell}{\pi} \ln \left[1+\frac{w}{a}+\sqrt{\left(1+\frac{w}{a}\right)^{2}-1}\right]$

Where $\varepsilon_{r}$ is the dielectric constant, $\varepsilon_{o}$ is the electric constant, $\ell$ is the length of the electrode, $w$ is the width of the electrode and $a$ is half gap between electrode. Equation (1) shows that the capacitance response depends on the dielectric and electric constant, and the geometry of electrode sensor. In this paper, the device development and the image reconstruction for stagnant and hydrodynamic samples using the device is discussed

\section{Design and Fabrication of 8-electrode Planar ECT}

The sensors of the miniaturized ECT are made by copper where the electrical conductive $(\sigma)$ is $5.69 \times 10^{7} \mathrm{~S} / \mathrm{m}$ at $20{ }^{\circ} \mathrm{C}$. Copper is relatively cheap as compared to other metal and it is easy to obtain and fabricate. The planar electrodes were fabricated on single sided PCB using the conventional PCB etching technique. The PCB layout was transferred using heat transfer machine. Later, ferric chloric acid is used to remove the unwanted copper layer. Fig. 2 indicates the configurations of the electrodes and measurement of the ECT planar electrodes.

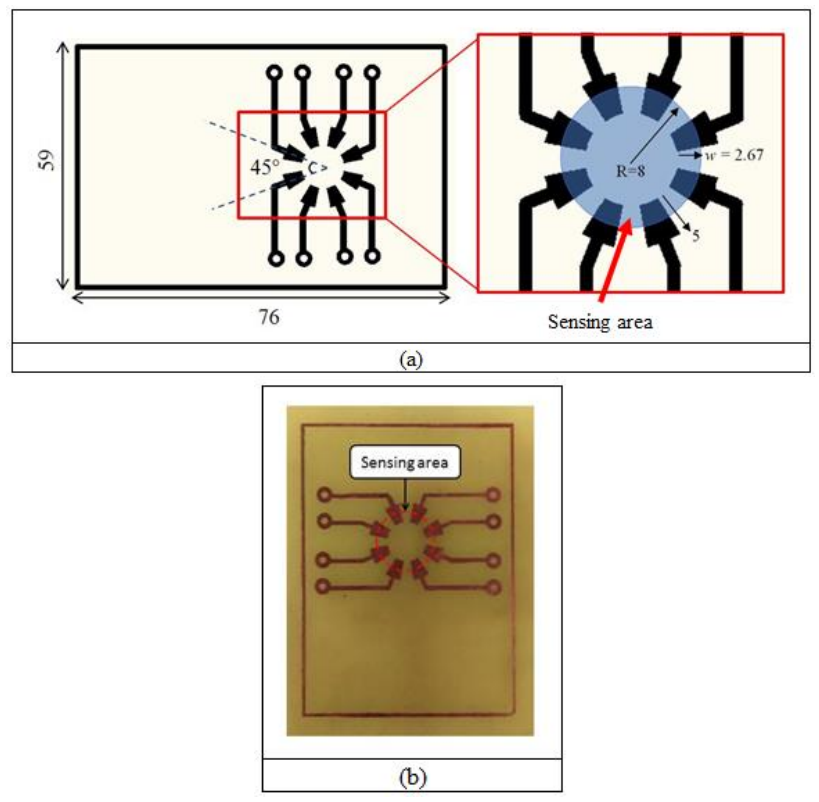

Fig. 2 8-planar electrode (a) dimension and arrangement and (b) fabricated electrodes

From Fig. 2(a), the overall size of the device is $76 \mathrm{~mm} \times 59 \mathrm{~mm}$ where an eight-copper-electrode array is fabricated on. The electrodes array with each electrode size $5.00 \mathrm{~mm} \times 2.08 \mathrm{~mm}$ is distributed equally on the sensing area with the interval angle between electrodes is $45^{\circ}$. The diameter of the sensing area is $16 \mathrm{~mm}$ and the length of the electrode exposed in the sensing area is $2.67 \mathrm{~mm}$. The fabricated 8-electrode planar array sensor is shown in Fig. 2(b) where the red dotted line indicates the placement of the sensing chamber.

The microchannel is developed for both stagnant and hydrodynamic flow channel. Fig. 3(a) shows the Polydimethylsiloxane (PDMS) chamber for stagnant sample and Fig. 3(b) shows the channel for hydrodynamic flow for the same 8-electrode ECT system. The development of micro the chamber and channel is important to hold and to flow two-phase sample into the sensing area. PDMS is used for the fabrication of the micro channel as it offers several beneficial characteristics such as biocompatible. In addition, it is transparent at optical frequencies where the state of samples in sensing area can be observed easily. The PDMS micro channel was fabricated using molding technique to create structure of the PDMS micro channel using the PDMS elastomer kits where the prepolymer 
resin and the curing agent are prepared with the ratio of 10:1 (w:w) and are mixed thoroughly. The mixture is then poured on a master replica template and cured in the oven at $60^{\circ} \mathrm{C}$ for 1 hour.
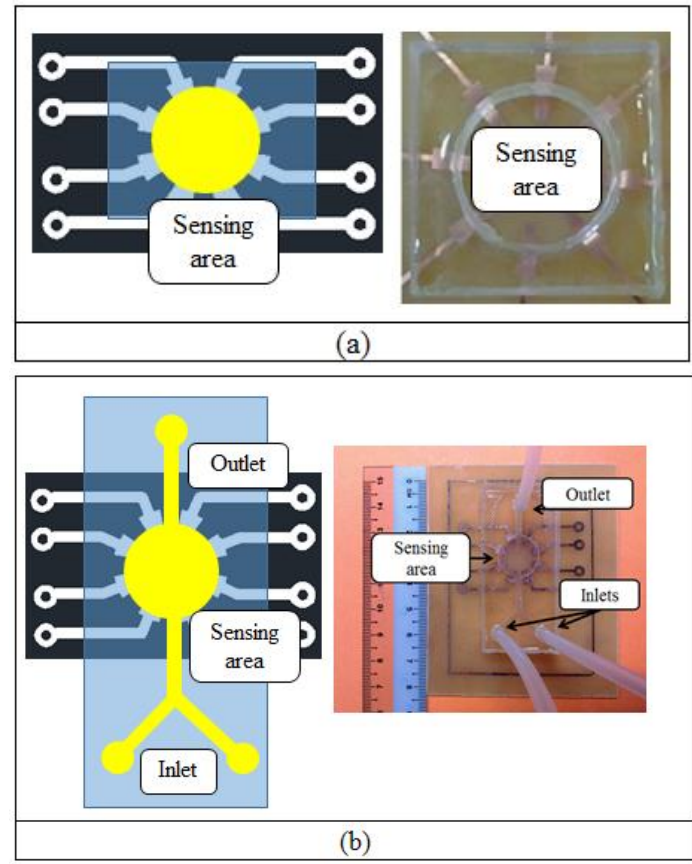

Fig. 3 PDMS chamber for (a) stagnant sample (b) hydrodynamic flow.

In Fig. 3, a $16 \mathrm{~mm}$ diameter PDMS chamber is the sensing area for both stagnant and hydrodynamic sample. For stagnant sample, the chamber is opened where the sample can be loaded directly into the chamber (Fig. 3(a)), whereas for the hydrodynamic sample, a conventional $\mathrm{Y}$-shape channel is used for transferring two fluid samples from the inlets into the enclosed sensing chamber. Same mixture of PDMS is prepared adhere the silicone tubes for the inlets and outlet. A very thin PDMS mixture is applied on the bonding area of PDMS channel and the bonding area of the planar electrodes. The chamber is aligned on the electrodes array and cure in the oven at $60^{\circ} \mathrm{C}$ for 1 hour.

For stagnant sample, the sample is placed on the sensing area, and capacitance values are measured. By using linear back projection (LBP) technique, the measured data is processed and image is reconstructed. For hydrodynamic flow, two fluid samples are delivered through the inlet by using micro pumps, the fluids are mixed and filled up in the chamber or the sensing area before exiting through the outlet. The image reconstruction for hydrodynamic flow is captured online as the flow changed.

The 8-electrode ECT planar is integrated with the hardware and software components to complete the system as shown in Fig. 4. The fan beam projection technique is used for the electrical configuration switching. The fan beam projection is selected for this experiment, where the transmitters and detectors can be alternately arranged until excitations of all electrodes are complete [6]. Thus, fan beam projection covers wider area of inspection and theoretically will produce better results of image reconstruction.

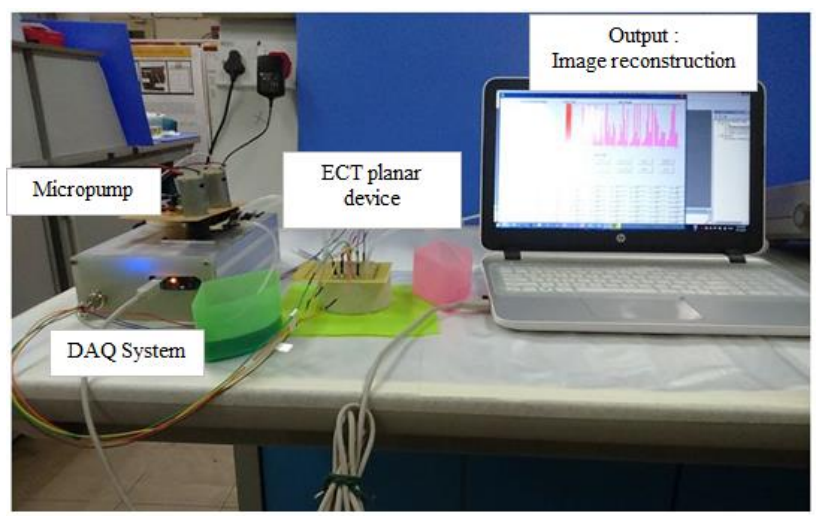

Fig. 4 Experimental set up for image reconstruction of two-phase fluid flow analysis.

The device is connected to the DAQ system for data collection and data processing. The data is sent to a control computer for image reconstruction. In addition, for hydrodynamic flow, a micro pump is used to supply the multiphase flow into the inlets and inspection chamber.

\section{Result and Discussion}

The image reconstruction is using LBP algorithm and $32 \times 32$ sensitivity map. The stagnant and hydrodynamic fluid within the sensing chamber is detected and reconstructed using the same planar ECT electrodes.

\subsection{Stagnant samples}

As for stagnant samples, the time taken for the detection and measurement process is not crucial and the image reconstruction can be done offline as the samples remain the same within the chamber as there is no movement or changes in the chamber. The image reconstruction results show that, various types of samples are easily reconstructed as shown in Fig. 5.

The stagnant samples consist of three different materials which are liquid - gas, liquid - liquid and solid - liquid. All three reconstructed images are able to resemble the real images from these combinations of materials which prove that the planar electrodes for the ECT device are able to reconstruct the images of these materials. The results are clearer for materials from different phase. Therefore a further study of the planar ECT system is by using hydrodynamic samples for liquid - liquid samples. 


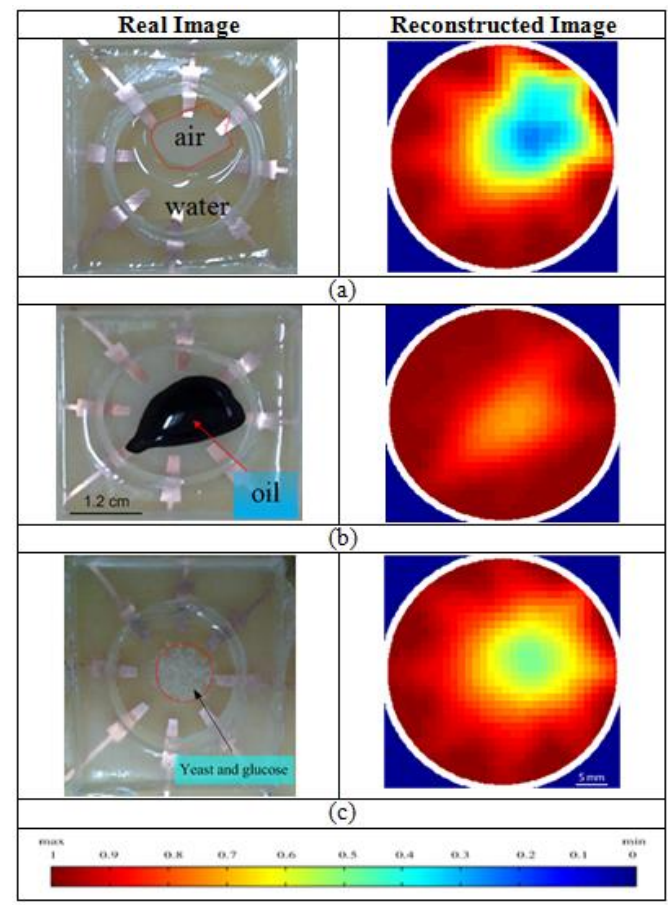

Fig. 5 Real and reconstructed images for stagnant samples (a) water-air (b) oil-water (c) yeast-glucose

\subsection{Hydrodynamic flow samples}

Hydrodynamic flow sample requires fast reconstruction algorithm for online monitoring. Liquidliquid samples tested the limit of the planar ECT device in distinguishing the fluids via this non-optical image reconstruction method. Fig. 6 and 7 show the image reconstructed for two liquid-liquid samples at constant flow rate $12.03 \mathrm{ml} / \mathrm{min}$.

\begin{tabular}{|c|c|c|}
\hline Times (s) & Reconstructed & Real \\
\hline 30 & oil $\quad$ water & oil water \\
\hline 60 & & \\
\hline 90 & & \\
\hline 120 & & \\
\hline
\end{tabular}

Fig. 6 Image reconstructions versus time at flow rate $12.03 \mathrm{ml} / \mathrm{min}$ for immiscible water -oil sample

\begin{tabular}{|c|c|c|}
\hline Times (s) & Reconstructed & Real \\
\hline 30 & & \\
\hline 60 & & \\
\hline 90 & & \\
\hline 120 & & \\
\hline
\end{tabular}

Fig. 7 Image reconstructions versus time at flow rate $12.03 \mathrm{ml} / \mathrm{min}$ for miscible water-glucose sample

From Fig. 6, the reconstructed images for immiscible liquid-liquid samples show good distinction between the oil and water within the sensing chamber even though within a flow regime. Whereas, the reconstructed images for miscible liquid-liquid of water (blue) and $0.31 \mathrm{~mol} / \mathrm{L}$ glucose solution (red) are not very clear (refer Fig. 7). However, the images shown are able to differentiate when one of the liquid is dominant in the sensing area; which is glucose.

\section{Conclusion}

The 8-electrode planar ECT developed able to detect the stagnant and hydrodynamic flow. For stagnant test, the time taking the data is an important variable as the sample stays at the sensing chamber. For stagnant sample, three experiments were conducted; water - air, oil - water and yeast - glucose. The results show that the ECT system able to detect the permittivity variation inside the sensing chamber.

For further study, the system is improved to test hydrodynamic flow measurements. For hydrodynamic flow, the data collection time is crucial to ensure the data collected is useful in flow monitoring. Thus, the experiment to capture image reconstructed versus time at flow rate $12.03 \mathrm{ml} / \mathrm{min}$ for immiscible water - oil sample, and miscible water - glucose solution is done. The system produces low quality image for miscible two multiphase flows, however, it is able to differentiate when glucose is dominating the sensor area. 


\section{References}

[1] M. S. Beck, T. Dyakowski, and R. A. Williams, "Process Tomography - The State of the Art," Transactions of the Institute of Measurement and Control, vol. 20, no. 4, pp. 163-177, 2016.

[2] M. Zhang, "Permittivity and Conductivity Imaging in Electrical Capacitance Tomography," $\mathrm{PhD}$, The Department of Electronic and Electrical Engineering, University of Bath, 2015.

[3] A. K. Allam and W. A. Deabes, "Electrical Capacitance Tomography Digital Processing Platform (ECT-DPU)," in IECON 2016 - 42nd Annual Conference of the IEEE Industrial Electronics Society, 2016, pp. 4767-4771.

[4] Z. Ren and W. Q. Yang, "A Miniature TwoPlate Electrical Capacitance Tomography Sensor," Ieee Sensors Journal, vol. 15, no. 5, pp. 3037-3049, May 2015.

[5] M. Mkandawire, "From Biosensors to Lab-OnChip: Developing Chip-Based Biosensors for Environmental and Clinical Applications," in 2012 5th International Conference on
Computers and Devices for Communication (CODEC), 2012, pp. 1-1.

[6] F. Y. N. Rahman et al., "A Review on Electrical Capacitance Tomography Sensor Development," Jurnal Teknologi, vol. 73, no. 3, pp. 35-41, 2015.

[7] Yasmin Abdul Wahab, Ruzairi Abdul Rahim, Mohd Hafiz Fazalul Rahiman, Suzanna Ridzuan Aw, Fazlul Rahman Mohd Yunus, Chiew Loon Goh, Herlina Abdul Rahim, Leow Pei Ling, "Non-invasive process tomography in chemical mixtures - A review ",Sensors and Actuators B: Chemical, Elsevier Sciences, (ISSN 0925-4005) vol. 210, Pp 602-617

[8] . Z, Mohd. Muji, R. Abdul Rahim, M. H. Fazalul Rahiman, S. Sahlan, M. F. Abdul Shaib , M. J. And E. J. Mohamad (July 2011), "Optical Tomography: A Review on Sensor Array, Projection Arrangement and Image Reconstruction Algorithm", International Journal of Innovative Computing, Information and Control", Tokai University, Vol. 7, No. 7, Pp. 1-17 